\title{
Relativistic Electron Precipitation Detections with CALET on the International Space Station
}

\author{
Alessandro Bruno, ${ }^{a, b, *}$ Lauren Blum, ${ }^{c}$ Georgia A. de Nolfo, ${ }^{a}$ Anthony W. Ficklin ${ }^{d}$ and \\ T. Gregory Guzik ${ }^{d}$ on behalf of the CALET Collaboration \\ (a complete list of authors can be found at the end of the proceedings) \\ ${ }^{a}$ Heliophysics Science Division, NASA Goddard Space Flight Center, Greenbelt, MD, USA \\ ${ }^{b}$ Department of Physics, Catholic University of America, Washington, DC, USA \\ ${ }^{c}$ University of Colorado, Boulder, CO, USA. \\ ${ }^{d}$ Department of Physics and Astronomy, Louisiana State University, Baton Rouge, LA, USA \\ E-mail: alessandro.bruno-1@nasa.gov
}

\begin{abstract}
The CALorimetric Electron Telescope (CALET) is a high-energy astroparticle physics experiment installed on the International Space Station, and taking data since October 2015. While designed for studying the origin and the propagation of galactic cosmic rays, CALET is also able to provide a continuous monitoring of space-weather phenomena affecting the near-Earth environment, including solar energetic particle and relativistic electron precipitation (REP) events. In this work we present preliminary results of the REP observations made over a $\sim 4.5$ year acquisition time (October 2015 - May 2020), investigating their correlations with the interplanetary and geomagnetic conditions. We also took advantage of a multi-spacecraft study using the twin Van Allen Probe measurements to complement CALET detections in low-Earth orbit, enabling a more complete picture of the global precipitation rates and drivers.
\end{abstract}

$37^{\text {th }}$ International Cosmic Ray Conference (ICRC 2021)

July 12 th - 23rd, 2021

Online - Berlin, Germany

\footnotetext{
*Presenter
} 

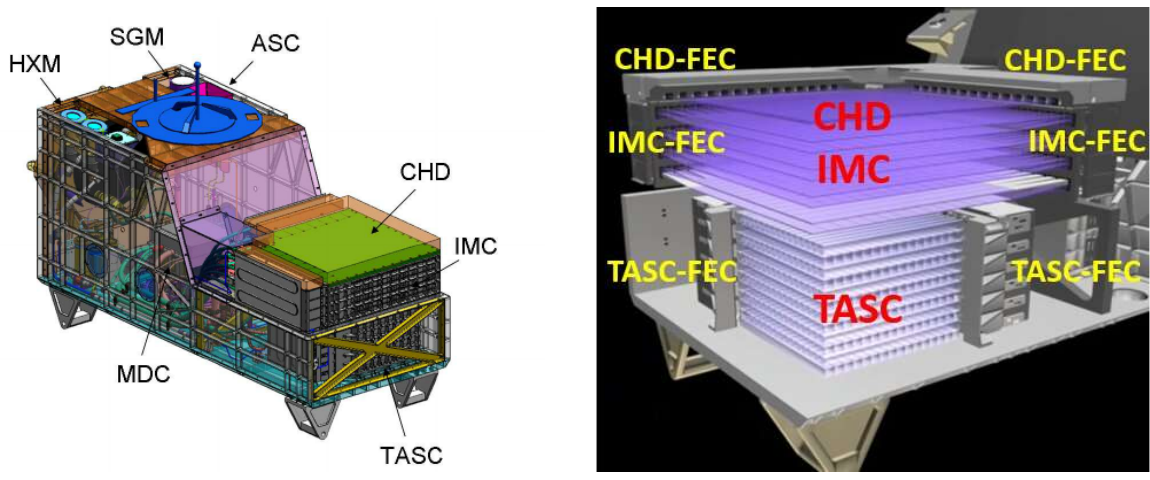

Figure 1: Left: schematic view of the CALET instrument showing the main calorimeter, the Gamma-ray Burst Monitor (CGBM) consisting of a hard X-ray monitor, and a soft gamma-ray monitor, Mission Data Controller (MDC) and support sensors, including Global Position Sensor Receiver (GPSR) and Advanced Stellar Compass (ASC) as indicated in the figure. Right: layout of the main calorimeter, which consists of a Charge Detector (CHD), an IMaging Calorimeter (IMC), and Total AbSorption Calorimeter (TASC), where FEC stands for front end electronics.

\section{Introduction}

Relativistic electron precipitation (REP) is a space-weather phenomenon commonly observed at high latitudes, in which energetic electrons trapped in the geomagnetic field are lost into the upper or middle atmosphere. It plays an important role in the magnetosphere dynamics, in particular during depletion intervals of the outer belt, and it has also a significant impact on the electrodynamics and chemical structure of the atmosphere. REP events are thought to be predominantly originated by pitch-angle scattering into the loss cone by plasma waves, field-line curvature scattering or loss through the magnetopause. Statistically, they occur more frequently during the the declining phase of the solar cycle, mostly in association with high-speed streams (HSSs) and under active geomagnetic conditions. In this work we present the preliminary REP observations with the CALorimetric Electron Telescope (CALET) on the International Space Station (ISS).

\section{The CALET experiment}

The CALET experiment is the result of an international collaboration involving Japan, Italy and USA [1]. Installed on the ISS in August 2015, it has been accumulating scientific data since October 2015. Its scientific goals include the investigation of nearby cosmic-ray accelerators and the search for dark matter, by precisely measuring all-electron $\left(\mathrm{e}^{-}\right.$and $\left.\mathrm{e}^{+}\right)$and gamma-ray spectra from $1 \mathrm{GeV}$ to $20 \mathrm{TeV}$, the energy spectra of protons, heliums, and heavier nuclei up to $\sim 1 \mathrm{PeV} /$ particle, and the ultra-heavy $(Z>28)$ nuclei composition above $600 \mathrm{MeV} / \mathrm{n}$.

A schematic overview of the instrument is shown in the left panel of Figure 1, with the main components given by the electromagnetic calorimeter, displayed in the right panel, and the gamma-ray burst monitor. The CALET calorimeter is a large-area high-performance instrument composed of a charge detector (CHD), a pre-shower imaging calorimeter (IMC) and a total absorption calorimeter (TASC). The CHD is a two-layer hodoscope of plastic scintillators providing the necessary charge resolution for the measurement of light and heavy nuclei. The IMC is a finely 

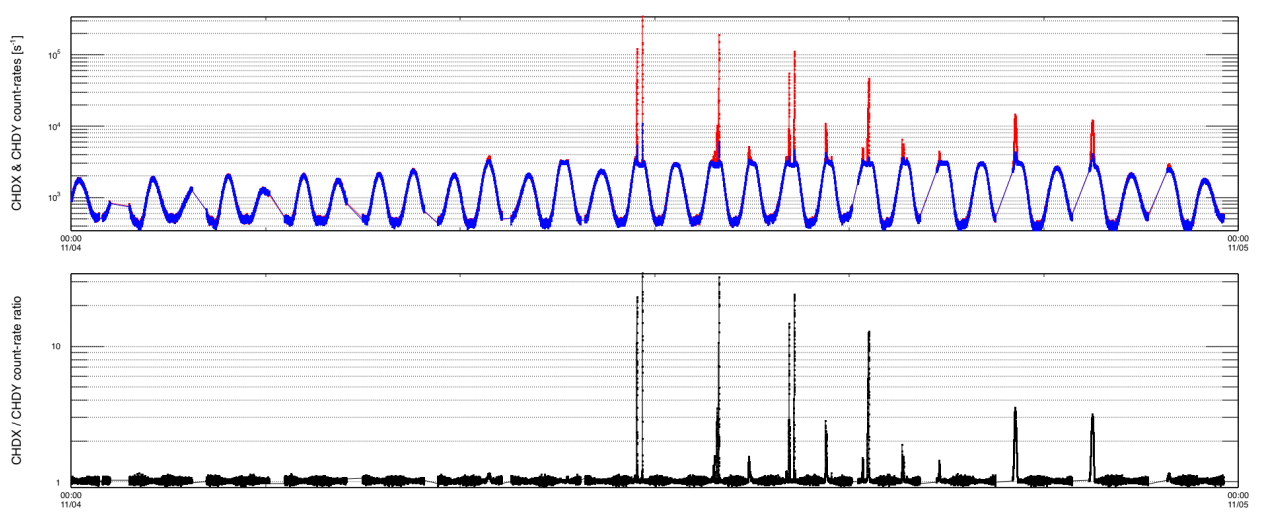

Figure 2: Top: temporal profile of the CHDX (red) and CHDY (blue) count rates during November 4, 2015. Bottom: corresponding count-rate ratio. The spikes associated with REP events are clearly visible.

segmented sampling calorimeter used to determine the incoming particle trajectory, supporting the measurement of the particle shower by the TASC. The latter is a homogeneous calorimeter with a 27 radiation-length thickness, which completely absorbs the electron shower energy in the TeV energy range and separate electrons from the overwhelming flux of protons. Details about the apparatus and its performance can be found elsewhere (e.g. [1,2] and references therein). By combining its unique identification capabilities and the long-term observations enabled by the installation on the ISS, CALET is expected to extend the limits of previous space-based experiments.

In addition to the aforementioned astrophysics goals, CALET is able to provide a continuous monitoring of the radiation environment in low-Earth orbit (LEO), including solar energetic particle events, inner-belt protons in the South Atlantic Anomaly (SAA) region, and REP events near the inner boundary of the outer radiation belt [3-6].

\section{Data analysis}

CALET REP observations rely on the count-rate information of the two CHD orthogonal layers, CHDX and CHDY, with a detection threshold corresponding to $\sim 1.5$ and $\sim 3.4 \mathrm{MeV}$ electrons, respectively. The present study is based on the dataset collected between October 2015 to May 2020, during the extended solar minimum phase between solar cycles 24 and 25 . The analyzed sample includes data collected at relatively high geomagnetic latitudes (Mcllwain's $L>2 \mathrm{R}_{E}$ ), excluding periods of high-energy SEP events (e.g., during early September 2017 [3]). As shown in Figure 2, REP events are recognizable as spikes in the CHD count rates. Displayed data do not include observations made in the SAA $\left(B<0.25 \mathrm{G}\right.$ and $\left.L<2.6 \mathrm{R}_{E}\right)$. Specifically, REP intervals were identified by requiring a count-rate ratio:

$$
R_{x y}=C H D X / C H D Y>1+3 \sigma_{R_{x y}},
$$

being $\sigma_{R_{x y}}$ the statistical uncertainty on $R_{x y}$. Overall, almost $3.8 \times 10^{4}$ REP intervals were selected. However, as discussed later, the sample includes a significant background component associated with the loss-cone electron population beneath the outer radiation belt, mostly detected in the geographic region South of the SAA. 

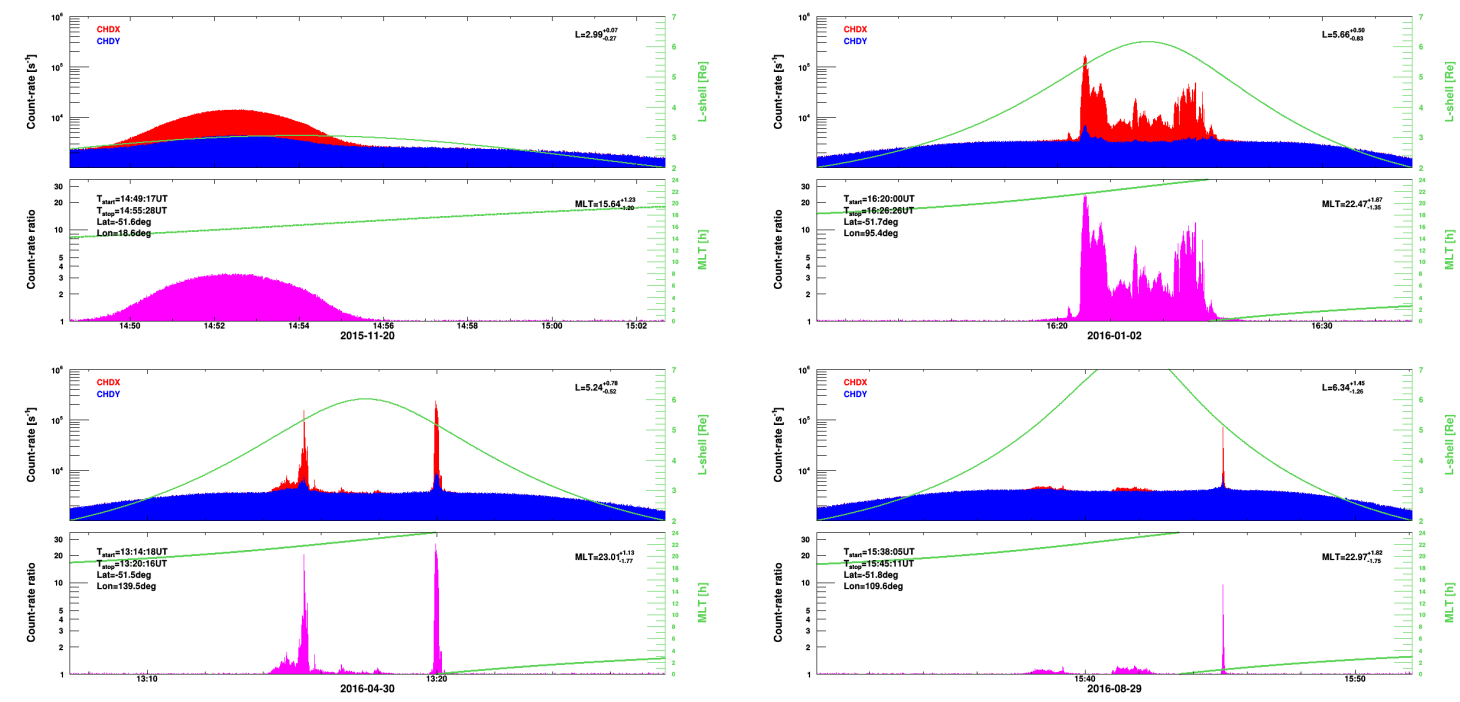

Figure 3: Four examples of REP intervals observed by CALET. The top panels show the temporal profiles of the CHDX (red) and CHDY (blue) count rates, while the bottom panels display the corresponding count-rate ratios (magenta). The two green curves indicate the $L$-shell (top) and the MLT (bottom) values, respectively.

Figure 3 reports four sample REP intervals observed by CALET, characterized by quite different duration and morphology. The top panels show the temporal profiles of the CHDX (red) and CHDY (blue) count rates, while the bottom panels display the corresponding count-rate ratio (magenta), which can be used to provide an estimate of the REP event spectral hardness. The two green curves indicate, respectively, the $L$-shell (top) and the magnetic local time (MLT, bottom) values along the orbit. The magnetic variables were estimated by using the International Geomagnetic Reference Field-13 (IGRF-13) [7] and the Tsyganenko \& Sitnov 2005 [8] models for the description of the internal and external geomagnetic field, respectively.

As expected, REP events were found to concentrate around the plasmapause, which is known to control particle distributions and dynamics in the inner magnetosphere. This is demonstrated in Figure 4, where the distribution of REP event detection drift shells relative to the plasmapause location $\left(L-L_{p p}\right)$ is shown as a function of MLT. Each point denotes the coordinates corresponding to the maximum CHDX count rate during the selected interval, with the color code indicating the count-rate ratio $R_{x y}$. For the description of the plasmapause we used the dynamical empirical model by [9]. In this case, for consistency with the model, the ISS magnetic coordinates were obtained by using the Tsyganenko 1989 model for the external geomagnetic field [10]. In line with the general trend of outer-belt electron fluxes, REP events with softest spectra (highest $R_{x y}$ values) were mainly found to populate the pre-midnight sector outside the plasmasphere. This is also demonstrated by the dial plots in Figure 5, where the magnetic latitude vs MLT distribution of the subsample corresponding to $R_{x y}>9$ (right panel) is compared to the one obtained with the full dataset (left panel). 


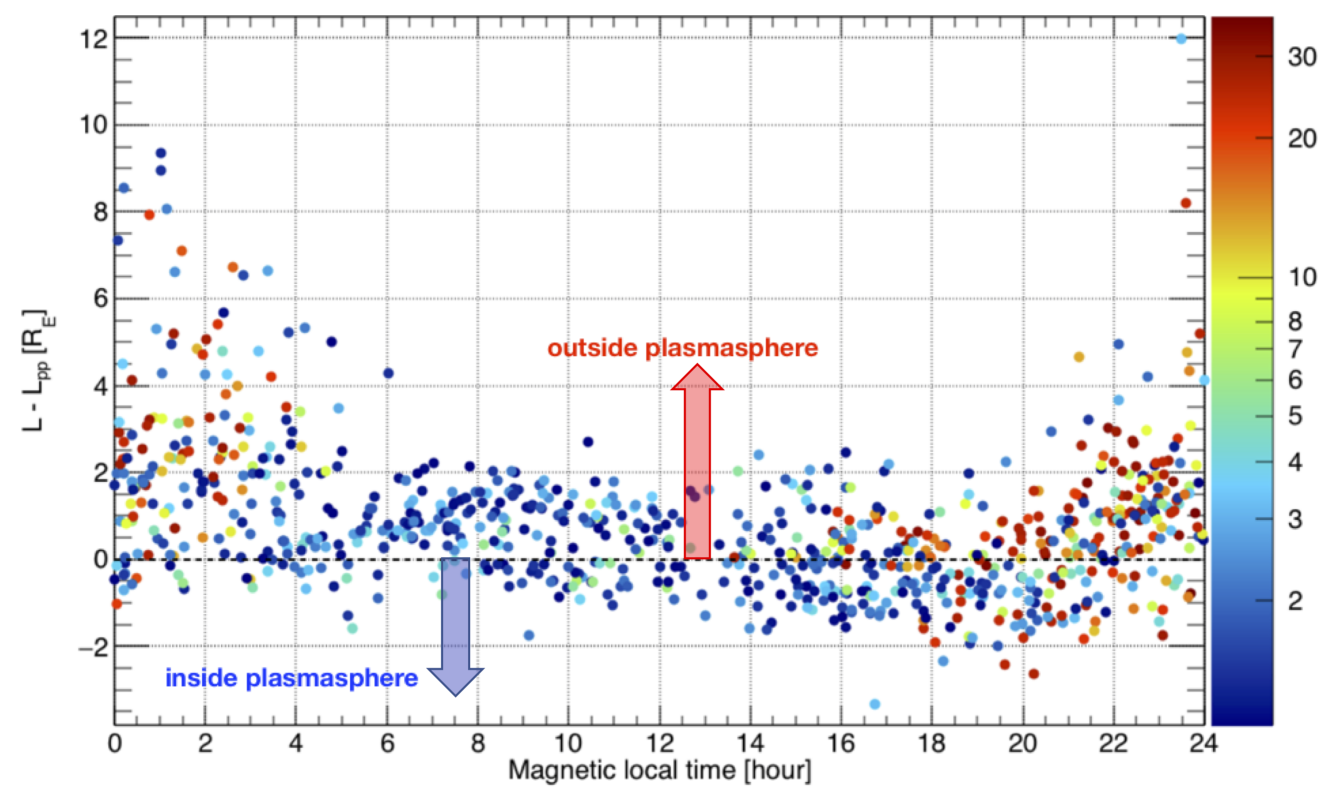

Figure 4: Distribution of REP event detection $L$-shell relative to the plasmapause $\left(L_{p p}\right)$, based on the empirical model by [9], as a function of MLT. The color code indicates the count-rate ratio $R_{x y}$.
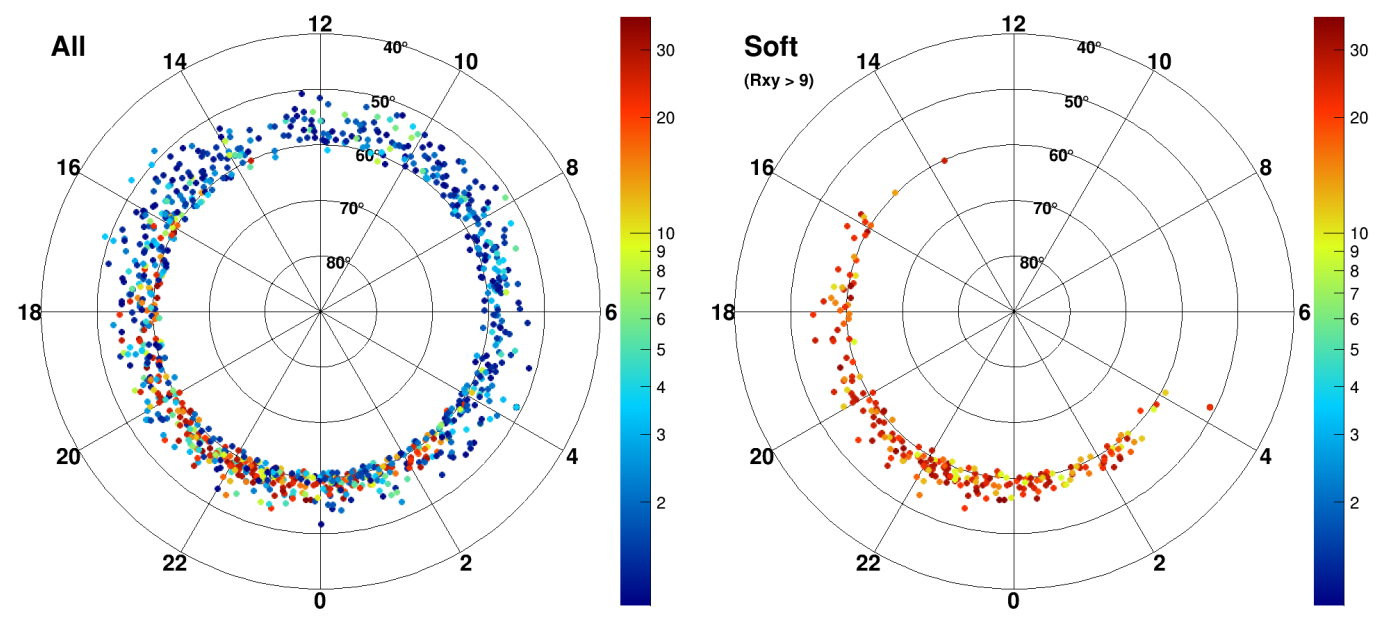

Figure 5: Left: spatial distribution of REP events as a function of magnetic latitude and local time, with the color code marking the maximum CHDX/CHDY count-rate ratio. Right: subsample of REP events with a maximum count-rate ratio greater than 9 .

\section{REP observations and contextual data}

To have a clearer view of the detected REP events, we compared CALET count rates to outer radiation-belt electron measurements and other contextual data, including interplanetary and geomagnetic parameters. For example, Figure 6 shows the analyzed observations as a function of time, during March 2017. Specifically, the temporal profiles of the CHDX and CHDY count rates and their ratio are shown in the two top panels. The third panel reports the color-coded electron intensity measured by the twin Van Allen Probes as a function of $L$-shell, at an energy (1.8 MeV) 


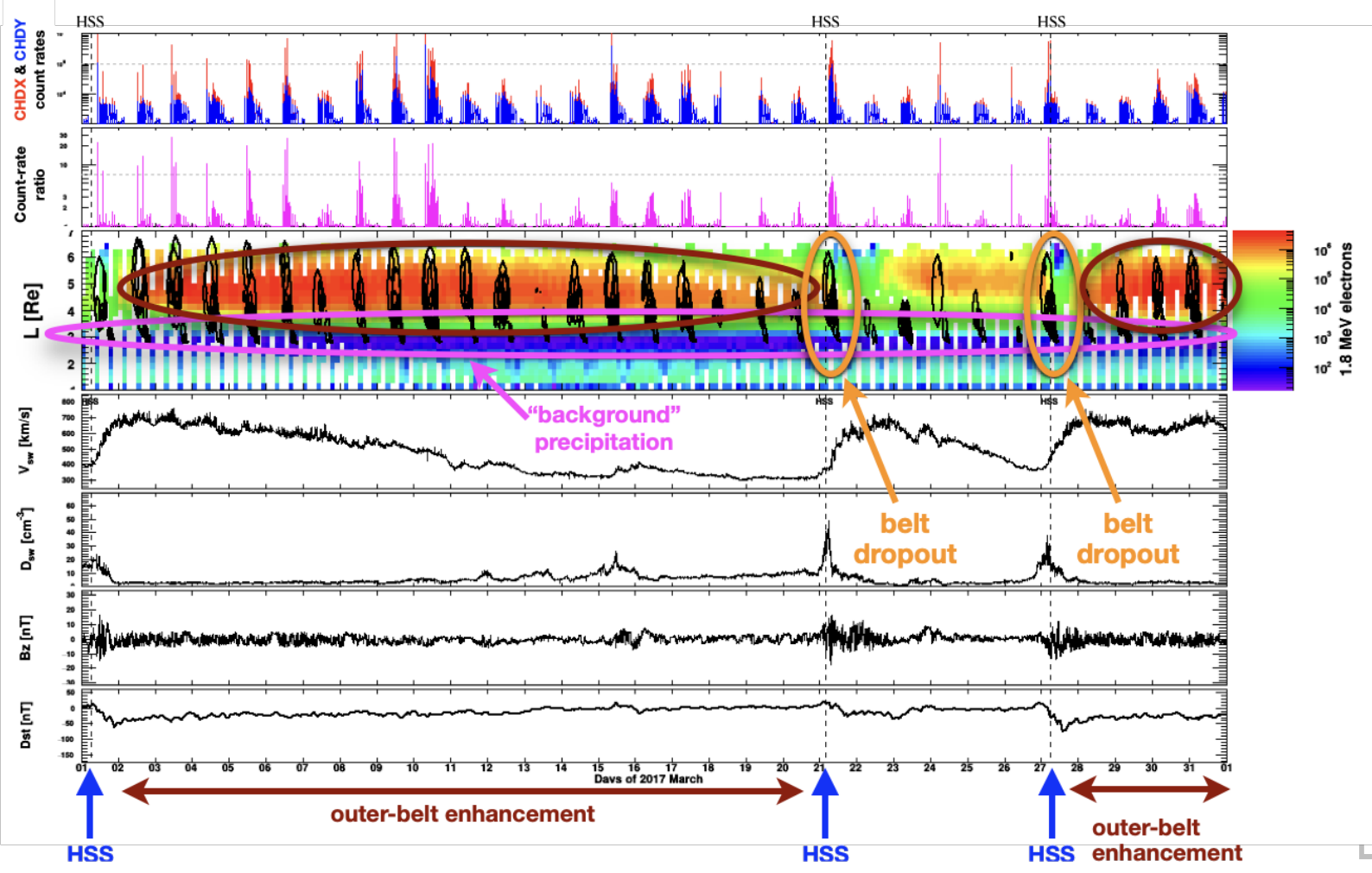

Figure 6: CALET REP observations and contextual data as a function of time, during March 2017. From top to bottom: CHDX and CHDY count rates, their ratio, the $1.8 \mathrm{MeV}$ electron flux measured by the Van Allen Probes (with the black ovals marking CALET detections), the solar wind speed and density, the IMF z-component and the Dst index. The three vertical dashed lines denote the arrival of HSSs.

similar to the CHDX electron threshold; the superimposed black ovals mark the CALET detections, with a size corresponding to the $L$-shell extension of the measured precipitation. The four bottom panels display the temporal profiles of the solar wind speed and density, the interplanetary magneticfield (IMF) z-component and the Dst geomagnetic index. Finally, the vertical lines mark the arrival of three HSSs, which are known to be important drivers of geomagnetic activity, influencing the outer-belt dynamics.

CALET REP observations can be classified into three categories. The first corresponds to events recorded during periods of enhanced outer-belt electron intensities, emphasized by the darkred ovals in Figure 6. The second comprises events detected during intervals of outer-belt depletions, typically following the arrival of solar-wind structures, like those marked by the orange ovals. The third category includes ordinary loss-cone electrons, commonly observed at relatively low latitudes around $L \sim 3 \mathrm{R}_{E}$, as indicated by the magenta oval. This precipitation is not necessarily linked to local scattering mechanisms, and constitutes a background component to REP events. While the CHD count rates of the first two classes often exhibit localized peaks or complex structures (see Figure 3), the events in the third category are typically characterized by a wide, smooth temporal profile of count rates, as shown in the top-left panel of Figure 3. 


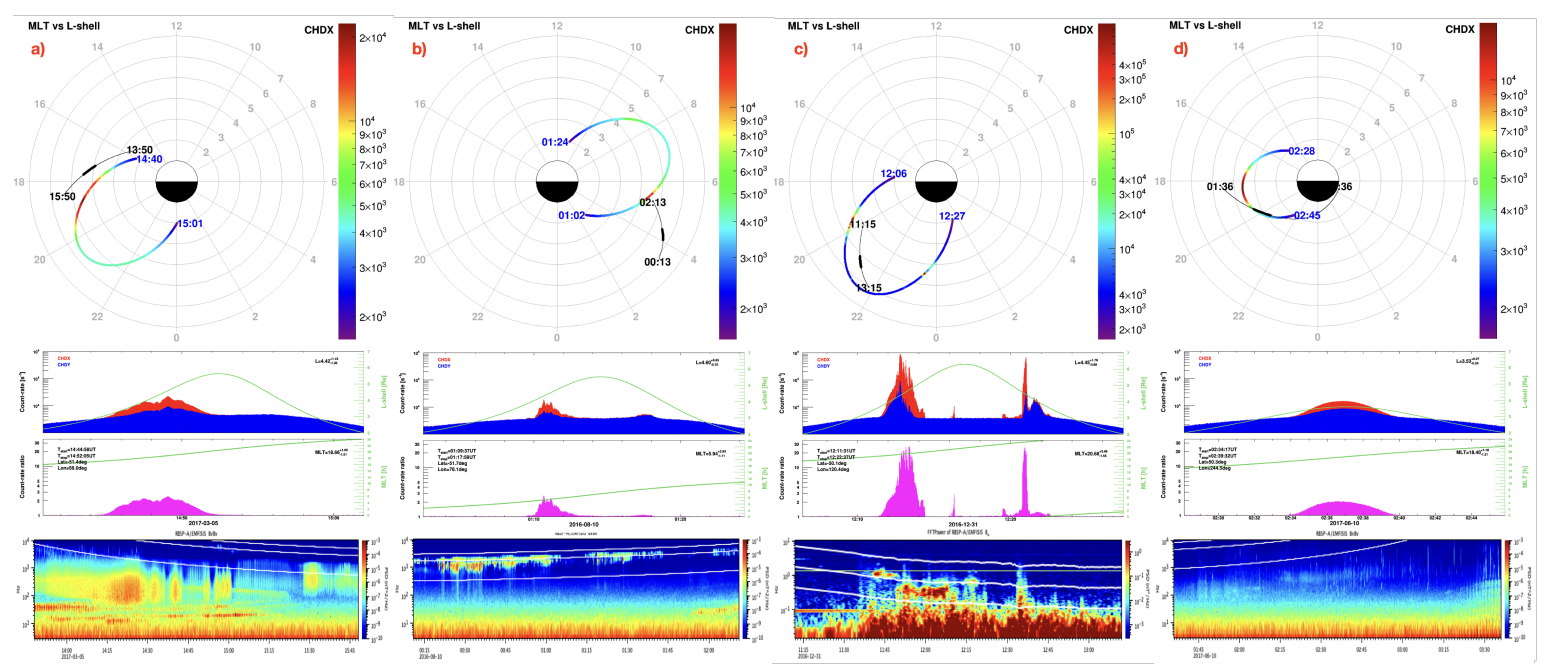

Figure 7: Sample conjunction events observed by CALET and the Van Allen Probes. See text for details.

\section{Coordinated study with the Van Allen Probes}

We took advantage of the plasma wave measurements of the Van Allen Probes in the equatorial plane to investigate the drivers of the $\mathrm{MeV}$ electron precipitation observed by CALET. A coordinated study is in progress based on the analysis of conjunction intervals during the time period in which both missions were operative (October 2015 - June 2019). Four sample events are reported in Figure 7. The MLT vs L dial plots in the the top panels display the trajectory of the ISS during the selected REP interval ( 20-min), with the color code indicating the CHDX count rate; for comparison, the black curve denotes the trajectory of one of the Probes during the 2-hour interval around CALET detection, with the latter marked by the thick segment. The middle panels display the CALET count rates and count-rate ratios (see Figure 3). Finally, the bottom panels show the magnetic-field measurements made by the Probes during the 2-hour interval around CALET detection. Such results suggest, for the first three events, an association with three different wave drivers, namely plasmaspheric Hiss waves (a), whistler-mode chorus waves (b) and electromagnetic ion cyclotron (EMIC) waves (c). On the other hand, the fourth event (d) is a typical loss-cone event commonly observed at relatively low drift shells, apparently not linked to any particular wave activity.

\section{Conclusions and Future Work}

The CALET experiment on the ISS is able to provide a continuous monitoring of space-weather phenomena affecting the near-Earth environment, including relativistic electron precipitation. Its observations in LEO can be used to complement those of the Van Allen Probes in the highly-eccentric orbit. We are carrying out a coordinated study between the two missions to identify the plasma wave populations generated near the magnetic equator which are potentially responsible for the electron precipitation directly observed by CALET. In addition, taking advantage of the large recorded data sample, we plan to perform a statistical investigation of REP occurrence vs solar-wind/geomagnetic drivers, in order to sort REP events by wave-driver and precipitation type (temporal profiles, spectral hardness, etc.), enabling a more complete picture of the global precipitation origin and rates. 


\section{Acknowledgments}

The authors gratefully thank Ian G. Richardson for the help in the analysis of solar-wind data.

\section{References}

[1] S. Torii, et al. 2016, The calorimetric electron telescope (CALET): a high-energy astroparticle physics observatory on the international space station, PoS ICRC2015, 236, 581, https: //doi.org/10.22323/1.236.0581

[2] O. Adriani, et al. 2019, Direct Measurement of the Cosmic-Ray Proton Spectrum from $50 \mathrm{GeV}$ to $10 \mathrm{TeV}$ with the Calorimetric Electron Telescope on the International Space Station, Phys. Rev. Lett. 122,181102, https://doi .org/10.1103/PhysRevLett.122.181102

[3] A. Bruno, et al. 2019, Space Weather Observations during September 2017 with CALET on the International Space Station, PoS ICRC2019, 1063, https://doi.org/10.22323/1. 358.1063

[4] R. Kataoka, et al. 2016, Relativistic electron precipitation at International Space Station: Space weather monitoring by Calorimetric Electron Telescope, Geophys. Res. Lett., 43, 4119, https://doi .org/10.1002/2016GL068930

[5] H. Ueno et al., 2020, Radiation Dose During Relativistic Electron Precipitation Events at the International Space Station, Space Weather, 18, https://doi.org/10.1029/ 2019Sw002280

[6] R. Kataoka, et al. 2020, Plasma Waves Causing Relativistic Electron Precipitation Events at International Space Station: Lessons From Conjunction Observations With Arase Satellite, J. Geophys. Res.: Space Physics, 125, e2020JA027875, https://doi .org/10.1029/ 2020JA027875

[7] P. Alken, et al. 2021, International Geomagnetic Reference Field: the thirteenth generation, Earth, Planets and Space, 73, 49, https: //doi .org/10.1186/s40623-020-01288-x

[8] N. A. Tsyganenko \& M. I. Sitnov, 2005, Modeling the dynamics of the inner magnetosphere during strong geomagnetic storms, J. Geophys. Res., 110, A03208, https://doi .org/10 . 1029/2004JA010798

[9] X. Liu, et al., 2015, Dynamic plasmapause model based on THEMIS measurements, J. Geophys. Res.: Space Physics, 120, 10,543, https://doi .org/doi : 10 . 1002/2015]A021801

[10] N. A. Tsyganenko, 1989, A magnetospheric magnetic field model with a wrapped tail current sheet, Planet. Space Sci., 37, 5, https://doi .org/10 . 1016/0032-0633(89)90066-4 


\section{Full Authors List: CALET Collaboration}

O. Adriani ${ }^{1,2}$, Y. Akaike ${ }^{3,4}$, K. Asano ${ }^{5}$, Y. Asaoka ${ }^{5}$, E. Berti1,2, G. Bigongiari 6,7 , W. R. Binns ${ }^{8}$, M. Bongi ${ }^{1,2}$, P. Brogi ${ }^{6,7}$, A. Bruno ${ }^{9,10}$, J. H. Buckley ${ }^{8}$, N. Cannady ${ }^{11,12,13}$, G. Castellini ${ }^{14}$, C. Checchia ${ }^{6}$, M. L. Cherry ${ }^{15}$, G. Collazuol ${ }^{16,17}$, K. Ebisawa ${ }^{18}$, A. W. Ficklin ${ }^{15}$, H. Fuke ${ }^{18}$, S. Gonzi ${ }^{1,2}$, T. G. Guzik ${ }^{15}$, T. Hams ${ }^{11}$, K. Hibino ${ }^{19}$, M. Ichimura ${ }^{20}$, K. Ioka $^{21}$, W. Ishizaki ${ }^{5}$, M. H. Israel ${ }^{8}$, K. Kasahara ${ }^{22}$, J. Kataoka ${ }^{23}$, R. Kataoka ${ }^{24}$, Y. Katayose ${ }^{25}$, C. Kato $^{26}$, N. Kawanaka ${ }^{27,28}$, Y. Kawakubo ${ }^{15}$, K. Kobayashi ${ }^{3,4}$, K. Kohri ${ }^{29}$, H. S. Krawczynski ${ }^{8}$, J. F. Krizmanic ${ }^{11,12,13}$ P. Maestro $^{6,7}$, P. S. Marrocchesi 6,7 , A. M. Messineo ${ }^{30,7}$, J.W. Mitchell ${ }^{12}$, S. Miyake ${ }^{32}$, A. A. Moiseev ${ }^{33,12,13}$, M. Mori ${ }^{34}$, N. Mori ${ }^{2}$, H. M. Motz ${ }^{35}$, K. Munakata ${ }^{26}$, S. Nakahira ${ }^{18}$, J. Nishimura ${ }^{18}$, G. A. de Nolfo ${ }^{9}$, S. Okuno ${ }^{19}$, J. F. Ormes ${ }^{36}$, N. Ospina ${ }^{16,17}$, S. Ozawa ${ }^{37}$, L. Pacini ${ }^{1,14,2}$, P. Papini ${ }^{2}$, B. F. Rauch ${ }^{8}$, S. B. Ricciarini ${ }^{14,2}$, K. Sakai ${ }^{11,12,13}$, T. Sakamoto ${ }^{38}$, M. Sasaki ${ }^{33,12,13}$, Y. Shimizu ${ }^{19}$, A. Shiomi ${ }^{39}$, P. Spillantini ${ }^{1}$, F. Stolzi 6,7 , S. Sugita ${ }^{38}$, A. Sulaj 6,7 , M. Takita ${ }^{5}$, T. Tamura ${ }^{19}$, T. Terasawa ${ }^{40}$, S. Torii ${ }^{3}$, Y. Tsunesada ${ }^{41}$, Y. Uchihori ${ }^{42}$, E. Vannuccini ${ }^{2}$, J. P. Wefel ${ }^{15}$, K. Yamaoka ${ }^{43}$, S. Yanagita ${ }^{44}$, A. Yoshida ${ }^{38}$, K. Yoshida ${ }^{22}$, and W. V. Zober ${ }^{8}$

${ }^{1}$ Department of Physics, University of Florence, Via Sansone, 1, 50019 Sesto, Fiorentino, Italy, ${ }^{2}$ INFN Sezione di Florence, Via Sansone, 1, 50019 Sesto, Fiorentino, Italy, ${ }^{3}$ Waseda Research Institute for Science and Engineering, Waseda University, 17 Kikuicho, Shinjuku, Tokyo 162-0044, Japan, ${ }^{4}$ JEM Utilization Center, Human Spaceflight Technology Directorate, Japan Aerospace Exploration Agency, 2-1-1 Sengen, Tsukuba, Ibaraki 305-8505, Japan, ${ }^{5}$ Institute for Cosmic Ray Research, The University of Tokyo, 5-1-5 Kashiwa-no-Ha, Kashiwa, Chiba 277-8582, Japan, ${ }^{6}$ Department of Physical Sciences, Earth and Environment, University of Siena, via Roma 56, 53100 Siena, Italy, ${ }^{7}$ INFN Sezione di Pisa, Polo Fibonacci, Largo B. Pontecorvo, 3, 56127 Pisa, Italy, ${ }^{8}$ Department of Physics and McDonnell Center for the Space Sciences, Washington University, One Brookings Drive, St. Louis, Missouri 63130-4899, USA, ${ }^{9}$ Heliospheric Physics Laboratory, NASA/GSFC, Greenbelt, Maryland 20771, USA, ${ }^{10}$ Department of Physics, Catholic University of America, Washington, DC 20064, USA, ${ }^{11}$ Center for Space Sciences and Technology, University of Maryland, Baltimore County, 1000 Hilltop Circle, Baltimore, Maryland 21250, USA, ${ }^{12}$ Astroparticle Physics Laboratory, NASA/GSFC, Greenbelt, Maryland 20771, USA, ${ }^{13}$ Center for Research and Exploration in Space Sciences and Technology, NASA/GSFC, Greenbelt, Maryland 20771, USA, ${ }^{14}$ Institute of Applied Physics (IFAC), National Research Council (CNR), Via Madonna del Piano, 10, 50019 Sesto, Fiorentino, Italy, ${ }^{15}$ Department of Physics and Astronomy, Louisiana State University, 202 Nicholson Hall, Baton Rouge, Louisiana 70803, USA, ${ }^{16}$ Department of Physics and Astronomy, University of Padova, Via Marzolo, 8, 35131 Padova, Italy, ${ }^{17}$ INFN Sezione di Padova, Via Marzolo, 8, 35131 Padova, Italy, ${ }^{18}$ Institute of Space and Astronautical Science, Japan Aerospace Exploration Agency, 3-1-1 Yoshinodai, Chuo, Sagamihara, Kanagawa 252-5210, Japan, ${ }^{19}$ Kanagawa University, 3-27-1 Rokkakubashi, Kanagawa, Yokohama, Kanagawa 221-8686, Japan, ${ }^{20}$ Faculty of Science and Technology, Graduate School of Science and Technology,, Hirosaki University, 3, Bunkyo, Hirosaki, Aomori 036-8561, Japan, ${ }^{21}$ Yukawa Institute for Theoretical Physics, Kyoto University, Kitashirakawa Oiwakecho, Sakyo, Kyoto 606-8502, Japan, ${ }^{22}$ Department of Electronic Information Systems, Shibaura Institute of Technology, 307 Fukasaku, Minuma, Saitama 337-8570, Japan, ${ }^{23}$ School of Advanced Science and Engineering, Waseda University, 3-4-1 Okubo, Shinjuku, Tokyo 169-8555, Japan, ${ }^{24}$ National Institute of Polar Research, 10-3, Midori-cho, Tachikawa, Tokyo 190-8518, Japan, ${ }^{25}$ Faculty of Engineering, Division of Intelligent Systems Engineering, Yokohama National University, 79-5 Tokiwadai, Hodogaya, Yokohama 240-8501, Japan, ${ }^{26}$ Faculty of Science, Shinshu University, 3-1-1 Asahi, Matsumoto, Nagano 390-8621, Japan, ${ }^{27}$ Hakubi Center, Kyoto University, Yoshida Honmachi, Sakyo-ku, Kyoto 606-8501, Japan, ${ }^{28}$ Department of Astronomy, Graduate School of Science, Kyoto University, Kitashirakawa Oiwake-cho, Sakyo-ku, Kyoto 6068502, Japan, ${ }^{29}$ Institute of Particle and Nuclear Studies, High Energy Accelerator Research Organization, 1-1 Oho, Tsukuba, Ibaraki 305-0801, Japan, ${ }^{30}$ University of Pisa, Polo Fibonacci, Largo B. Pontecorvo, 3, 56127 Pisa, Italy, ${ }^{31}$ Astroparticle Physics Laboratory, NASA/GSFC, Greenbelt, Maryland 20771, USA, ${ }^{32}$ Department of Electrical and Electronic Systems Engineering, National Institute of Technology, Ibaraki College, 866 Nakane, Hitachinaka, Ibaraki 312-8508, Japan ${ }^{33}$ Department of Astronomy, University of Maryland, College Park, Maryland 20742, USA, ${ }^{34}$ Department of Physical Sciences, College of Science and Engineering, Ritsumeikan University, Shiga 525-8577, Japan, ${ }^{35}$ Faculty of Science and Engineering, Global Center for Science and Engineering, Waseda University, 3-4-1 Okubo, Shinjuku, Tokyo 169-8555, Japan, ${ }^{36}$ Department of Physics and Astronomy, University of Denver, Physics Building, Room 211, 2112 East Wesley Avenue, Denver, Colorado 80208-6900, USA, ${ }^{37}$ Quantum ICT Advanced Development Center, National Institute of Information and Communications Technology, 4-2-1 Nukui-Kitamachi, Koganei, Tokyo 184-8795, Japan, ${ }^{38}$ College of Science and Engineering, Department of Physics and Mathematics, Aoyama Gakuin University, 5-10-1 Fuchinobe, Chuo, Sagamihara, Kanagawa 252-5258, Japan, ${ }^{39}$ College of Industrial Technology, Nihon University, 1-2-1 Izumi, Narashino, Chiba 275-8575, Japan ${ }^{40}$ RIKEN, 2-1 Hirosawa, Wako, Saitama 351-0198, Japan, ${ }^{41}$ Division of Mathematics and Physics, Graduate School of Science, Osaka City University, 3-3-138 Sugimoto, Sumiyoshi, Osaka 558-8585, Japan, ${ }^{42}$ National Institutes for Quantum and Radiation Science and Technology, 4-9-1 Anagawa, Inage, Chiba 263-8555, Japan, ${ }^{43}$ Nagoya University, Furo, Chikusa, Nagoya 464-8601, Japan, ${ }^{44}$ College of Science, Ibaraki University, 2-1-1 Bunkyo, Mito, Ibaraki 310-8512, Japan 